\title{
Body Imaging: Thorax and Abdomen. Anatomical Landmarks, Image Findings, Diagnosis
}

\author{
Gabriele A. Krombach, Andreas H. Mahnken \\ Thieme Stuttgart, New York, Delhi, Rio de Janeiro, 2018 \\ 497 pages, ISBN 978-3-13-205411-0
}

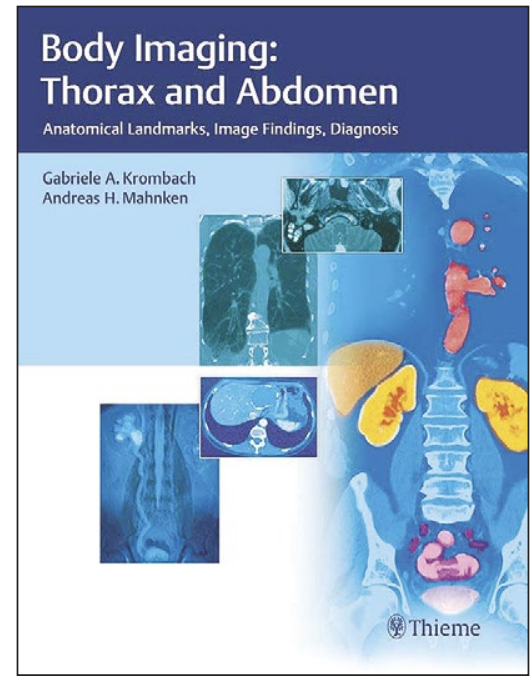

This textbook represents a valuable reference in the dynamic and challenging field of diagnostic and therapeutic imaging. According to its authors, the book is intended for those less experienced in of body imaging, being a practical and easy documentation resource for learning and updating knowledge.

The book is divided in two main parts, chest and abdomen, across 13 concise chapters. Each chapter is broken down into multiple sections including but not limited to anatomy, physiology and pathophysiology, imaging, anatomical variants, acquired diseases. Various disorders are systematized in a practical way offering relevant data about anatomy, clinical features, imaging signs, differential diagnosis and key points. The description of the most important anatomic landmarks is clear providing a rapid and confident orientation. The images and text are combined in a logic manner, providing a useful stepby-step diagnosis algorithm. The key points are summarized in very clear tables and boxes.

The first chapter focuses on the mediastinum, illustrating diffuse diseases, the masses and provides relevant images for trauma with mediastinal injury. The second chapter details the most important heart and pericardium diseases. Large vessels' pathology (congenital anomalies and specific disorders for aorta, vena cava, pulmonary vessels) is explained by drawings and $3 \mathrm{D}$ reconstruction CT images, making the classical radiographs easier to understand. Disorders of the lung and pleura are richly illustrated in chapter four. Specific liver pathology features depicted by ultrasound, computer tomography (CT), magnetic resonance imaging (MRI) are presented in chapter five. Benign and malignant diseases of the gallbladder, biliary tract and pancreas are discussed and illustrated in chapters six and seven. An attractive approach is proposed for the gastrointestinal tract pathology through CT and MRI with contrast media in chapter eight. The most relevant radiological images for the spleen, lymphatic tissue and adrenal glands diseases are presented in chapters nine and ten. Specific urology issues are illustrated in chapter eleven: masses, inflammatory changes, vascular diseases, renal transplant. The last two chapters are focused on female pelvis and male pelvis pathology.

Most important for the field of radiology are the numerous images throughout the book - more than 1600 figures with excellent resolution. Included in the book are sectional images from multiple modalities including ultrasonography, radiography, CT scan and MRI. These high quality and clearly labeled images are supplemented with explanatory drawings, diagrams and tables showing details of anatomy and pathology.

This book is an important tool also for clinicians in different specialties as it explains for a set of given symptoms which imaging modality is preferred, how are abnormalities recognized, what are the differential diagnoses to consider and what are the arguments for the final diagnosis.

Overall, this textbook is a high-quality resource not only for radiologists, but also for related specialties. The outline organizational structure, clear, pertinent imaging, and concise chapters recommend the book as an important diagnostic tool for clinical practice.

Ioana Călian

Cluj-Napoca, Romania 